\title{
WHEN MATRICES COMMUTE
}

\author{
TERRY A. LORING
}

\begin{abstract}
.
Necessary and sufficient conditions are given for certain almost commuting triples of matrices to be close to a commuting triple. Generalizations are given for triples in $C^{*}$-algebras that have sufficiently many invertible elements.
\end{abstract}

Lin gave the first proof of the following theorem.

Theorem 1 ([Lin1]). For any $\epsilon>0$, there is a $\delta>0$ so that, for any $d$ and any $h_{1}$ and $h_{2}$ in $\mathbf{M}_{d}$ satisfying

$$
\left\|h_{1} h_{2}-h_{2} h_{1}\right\| \leq \delta \text { and } 0 \leq h_{i} \leq 1,
$$

there exist hermitian elements $k_{1}$ and $k_{2}$ in $\mathbf{M}_{d}$ such that

$$
k_{1} k_{2}=k_{2} k_{1} \text { and }\left\|h_{i}-k_{i}\right\| \leq \epsilon .
$$

Subsequently, Friis and Rørdam [FR] gave a short proof of a stronger result that applies to almost normal elements in a broad class of $C^{*}$-algebras.

\section{Reduction to Lifting.}

In Lin's proof, as well as in the proof of Friis and Rørdam, the first step is a reduction to the problem of showing a very restricted form of semiprojectivity for $C(\mathbf{D})$. That is, one reduces to showing that one may approximately solve lifting problems of the form

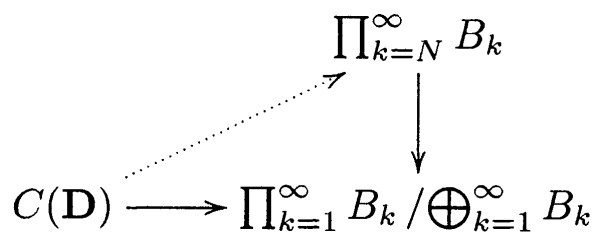

This research was partially supported by the National Science Foundation, DMS 9215024.

Received February 28, 1996. 
Friis and $\mathrm{R} \varnothing \mathrm{rdam}$ then show that enough maps have a specialized form of corona extendibility to reduce to the case where $\varphi$ factors through $C(X)$ and $X$ is a finite graph. The semiprojectivity (unrestricted, see [Lo2]) of $C(X)$ then produces the needed lifts.

This note examines what happens when $C(\mathbf{D})$ is replaced by $C\left(S^{2}\right)$. The most dramatic change is that a $K$-theoretic obstruction appears.

Let $\mathscr{B}$ denote a class of unital $C^{*}$-algebras, to be specified more precisely later.

The definition of boundedness of a set of relations $\mathscr{R}$ is that they determine a universal $C^{*}$-algebra $C^{*}\langle G \mid \mathscr{R}\rangle$. A $\delta$-representation of a set of relations of the form $\left\|p_{j}\left(x_{1}, \ldots, x_{l}\right)\right\| \leq \eta_{j}$ is a tuple $\left(z_{1}, \ldots, z_{l}\right)$ such that $\left\|p_{j}\left(z_{1}, \ldots, z_{l}\right)\right\| \leq \eta_{j}+\delta$. For present purposes, $p_{j}$ should be thought of as a polynomial in the $x_{k}, x_{k}^{*}$, and perhaps $f\left(\left|x_{k}\right|\right)$ for some bounded continuous function.

Definition 2. A set of finite, bounded relations $\mathscr{R}$ is weakly stable with respect to $\mathscr{B}$ if for every $\epsilon>0$ there is a $\delta>0$ such that, for every $\delta$-representation $x_{1}, \ldots, x_{l}$ of $\mathscr{R}$ in any $B \in \mathscr{B}$ there is a representation $y_{1}, \ldots, y_{l}$ of $\mathscr{R}$ in $B$ such that $\left\|y_{j}-x_{j}\right\| \leq \epsilon$ for $j=1, \ldots, l$.

Definition 3. A unital $C^{*}$-algebra $A$ is weakly semiprojective with respect to $\mathscr{B}$ if, for any sequence $B_{n} \in \mathscr{B}$, any unital $*$-homomorphism

$$
\varphi: A \rightarrow \prod B_{n} / \bigoplus B_{n}
$$

any $\epsilon>0$, and any finite set $G$ in $A$, there exists $m$ and a unital *-homomorphism

$$
\bar{\varphi}: A \rightarrow \prod_{n=m}^{\infty} B_{n}
$$

so that

$$
\left\|\rho_{m} \circ \bar{\varphi}(g)-\varphi(g)\right\| \leq \epsilon \quad(\forall g \in G)
$$

where $\rho_{m}$ is defined by

$$
\rho_{m}\left(\left(b_{m}, b_{m+1}, \ldots\right)\right)=\left(0, \ldots, 0, b_{m}, b_{m+1}, \ldots\right)+\bigoplus B_{n} .
$$

The following is known in special cases. See [Lin1] for the proof in the case $\mathscr{R}=\left\{\|x\| \leq 1 \& x^{*} x=x x^{*}\right\}$. The same proof works in general.

Theorem 4. For $A=C^{*}\langle G \mid \mathscr{R}\rangle$, with $\mathscr{R}$ a finite, bounded set of relations, the following are equivalent. 
(1) $\mathscr{R}$ is weakly stable w.r.t. $\mathscr{B}$.

(2) $\tilde{A}$ is weakly semiprojective w.r.t. $\mathscr{B}$

(3) for any sequence $B_{n} \in \mathscr{B}$, any unital $*$-homomorphism

$$
\varphi: A \rightarrow \prod B_{n} / \bigoplus B_{n},
$$

any $\epsilon>0$ there exists $m$ and a unital $*$-homomorphism

$$
\bar{\varphi}: A \rightarrow \prod_{n=m}^{\infty} B_{n}
$$

so that $\rho_{m} \circ \bar{\varphi}=\varphi$.

Recall from [Bl] that semiprojectivity for a unital $C^{*}$-algebra $A$ means that for any unital $\varphi: A \rightarrow C / J$, if $J$ is the closed union of an increasing chain $J_{k} \subseteq J_{k+1}$ of ideals in $C$ then there exists $n$ and a unital lift of $\varphi$ to $\bar{\varphi}: A \rightarrow C / J_{n}$. Therefore, semiprojectivity for $A$ implies weak semiprojectivity w.r.t. $\mathscr{B}$.

\section{Puncturing a sphere.}

Definition 5. A unital $*$-homomorphism $\eta: A_{1} \rightarrow A_{2}$ is weakly corona extendible with respect to $\mathscr{B}$ if, given a unital $*$-homomorphism

$$
\varphi: A_{1} \rightarrow \prod B_{n} / \bigoplus B_{n} \quad\left(B_{n} \in \mathscr{B}\right)
$$

there exists a unital $*$-homomorphism

$$
\gamma: A_{2} \rightarrow \prod B_{n} / \bigoplus B_{n}
$$

with $\gamma \circ \eta=\varphi$.

If $\mathscr{B}$ equals $\left\{\mathbf{M}_{d} \mid d \in \mathbf{N}\right\}$ this property is called "matricial corona extendibility."

It should be clear that the maps that are weakly corona extendible w.r.t. $\mathscr{B}$ are closed under composition.

We now specify the classes $\mathscr{B}$ of $C^{*}$-algebras that are relevant. A familiar class is the collection of $C^{*}$-algebras of stable rank one. We remind the reader this means that $G L(A)$ is dense in $A$, so in particular $\mathbf{M}_{d}$ has stable rank one. A larger class where our results hold is the class of $C^{*}$-algebras that have the property (IR) introduced by Friis and Rørdam.

For any unital $C^{*}$-algebra $A$, let $R(A)$ denote the set of elements $x$ such that, for every ideal $I$ of $A, \dot{x}$ one-sided invertible in $A / I$ implies $\dot{x}$ invertible in $A / I$. We say $A$ has property (IR) if every element of $R(A)$ is the norm 
limit of invertible elements of $A$. If $A$ is not unital, it is said to have property (IR) if $\tilde{A}$ does.

Gracious thanks are due to Søren Eilers for pointing out the errors in the early versions of this paper. Part (4) of the following suggested itself in the course of tracking down those errors. Additional thanks go to Mikael Rørdam for helpful communications regarding property (IR).

Lemma 6. Suppose $B$ is a unital $C^{*}$-algebra. Suppose $A_{1}, A_{2}, \ldots$ are unital (IR) $C^{*}$-algebras and let

$$
Q=\prod A_{n} / \bigoplus A_{n}
$$

(1) (Proposition 4.2 (i) of [FR]) For all $a$ in $B$, $\operatorname{dist}(a, R(B)) \leq\left\||a|-\left|a^{*}\right|\right\|$.

(2) If $x$ in $Q$ is normal then there exists a unitary $u$ in $Q$ such that $x=u|x|$.

(3) For any contractions $a$ and $b$ in $B$, and for $\delta \in[0,1 / 4)$, if

$$
\left\|a^{*} a+b^{*} b-1\right\| \leq \delta \text { and }\left\|\mathrm{aa}^{*}+\mathrm{bb}^{*}-1\right\| \leq \delta
$$

then $\operatorname{dist}(a, R(B)) \leq \sqrt{2 \delta}$.

(4) If $a$ in $Q$ is a contraction and there is another contraction $b$ in $Q$ such that

$$
a^{*} a+b^{*} b=1=a a^{*}+b b^{*}
$$

then there exists a unitary $u$ in $Q$ such that $a=u|a|$.

Proof. Friis and Rørdam prove (1) and use it to derive a result that is very similar to (2). Given (1), we may prove (2) as follows. Any normal element $x$ in $Q$ is the image of a sequence $\left(x_{n}\right)$ with $x_{n}$ in $A_{n}$ and

$$
||\left|x_{n}\right|-\left|x_{n}^{*}\right||| \rightarrow 0 \text {. }
$$

By (1) and the assumption that each $A_{n}$ is (IR), there is a sequence $y_{n}$ of invertible elements, each $y_{n}$ in $A_{n}$, with $\left\|x_{n}-y_{n}\right\| \rightarrow 0$. Since every invertible element in $A_{n}$ has a unitary polar decomposition in $A_{n}$, so must the image of $\left(y_{n}\right)$ in $Q$, which is $x$.

An almost identical argument shows that (3) implies (4).

Assume $a$ and $b$ are contractions in $B$ and

$$
\left\|a^{*} a+b^{*} b-1\right\| \leq \delta \quad \text { and } \quad\left\|a a^{*}+b b^{*}-1\right\| \leq \delta .
$$

For some partial isometry in $B^{* *}$, we have $a=v|a|$. Let

$$
a^{\prime}=v f(|a|)
$$

where $f:[0, \infty) \rightarrow[0, \infty)$ is continuous, sends $[0, \sqrt{2 \delta}]$ to $\{0\}$ and $|f(\lambda)-\lambda|$ is everywhere less than $\sqrt{2 \delta}$. Certainly $\left\|a-a^{\prime}\right\|=2 \delta$ and $a^{\prime} \in B$. We'll show 
$a^{\prime} \in R(B)$. For starters, we'll prove that $0 \in \sigma\left(\left|a^{\prime}\right|\right)$ if and only if $0 \in \sigma\left(\left|a^{\prime *}\right|\right)$. We'll break this into three cases: $a$ is invertible; $0 \in \sigma(|a|)$; and $0 \in \sigma\left(\left|a^{*}\right|\right)$. The symmetry in the roles of $a$ and $a^{*}$ allows us to consider only the first two cases.

If $a$ is invertible, then the spectrum of $a^{*} a$ equals the spectrum of $a a^{*}$. The spectra of $\left|a^{\prime}\right|$ and $\left|a^{\prime *}\right|$ must also be equal because we have

$$
\left|a^{\prime}\right|=f(|a|) \quad \text { and } \quad\left|a^{\prime *}\right|=f\left(\left|a^{*}\right|\right) .
$$

Suppose 0 is in $\sigma(|a|)$. Then 0 is in $\sigma\left(a^{*} a\right)$ and so the intersection of $[1-\delta, 1]$ with $\sigma\left(b^{*} b\right)$ is nonempty. Away from 0 the spectra of $b^{*} b$ and $b b^{*}$ agree, so $\sigma\left(b b^{*}\right) \cap[1-\delta, 1] \neq \emptyset$. Therefore, the spectrum of $a^{*} a$ contains some $\lambda_{0}$ with $0 \leq \lambda_{0} \leq 2 \delta$. By the way $f$ was defined, $f\left(\sqrt{\lambda_{0}}\right)=0$. The spectral theorem and (6) tell us that 0 is in the spectra of both $\left|a^{\prime}\right|$ and $\left|a^{\prime *}\right|$.

The inequalities in (5) and the equalities in (6) pass to quotients. Therefore the previous paragraph shows that that $a$ is in $R(Q)$.

Let $X_{1}$ denote the space

$$
X_{1}=\left\{r e^{2 \pi i \theta} \mid 1 \leq r \leq 2\right\}
$$

and let $\zeta: X_{1} \rightarrow \mathbf{D}$ be the map that sends $r e^{2 \pi i \theta}$ to $(r-1) e^{2 \pi i \theta}$. Define $\eta: C(\mathbf{D}) \rightarrow C\left(X_{1}\right)$ by

$$
\eta(f)=f \circ \zeta .
$$

Part (2) of the Lemma 6 is equivalent to the statement that $\eta$ is weakly corona extendible w.r.t. the unital (IR) $C^{*}$-algebras. (Notice that whenever a normal $x$ has a unitary polar decomposition $x=u|x|$, it follows that $u$ commutes with $|x|$.)

Proposition 7. If $U$ is a open set in $X_{1}$ that is disjoint from the circle $r=1$ then the $*$-homomorphism

$$
C(\mathbf{D} \backslash \zeta(U)) \rightarrow C\left(X_{1} \backslash U\right) .
$$

induced by $\zeta$ is weakly corona extendible w.r.t. any collection of $C^{*}$-algebras whose elements have property (IR).

Proof. There is a commutative diagram,

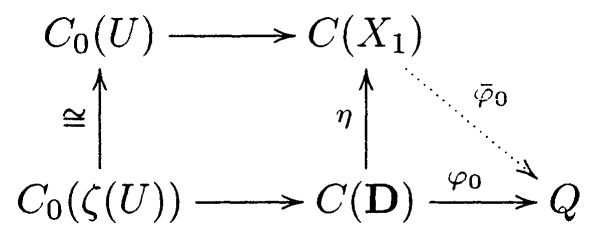


where $\varphi_{0}$ is the composition of the surjection of $C(\mathbf{D})$ onto $C(\mathbf{D} \backslash \zeta(U))$ with $\varphi$, and $\bar{\varphi}_{0}$ is the extension guaranteed by Lemma 6 . Since $\bar{\varphi}_{0}$ must send $C_{0}(U)$ to $\varphi_{0}\left(C_{0}(\zeta(U))\right)=0$, this drops to the quotient $C_{0}\left(X_{1} \backslash U\right)$.

We now introduce some more notation. Let $X_{n}$ denote the closed disk with $n$ disjoint open discs removed from the interior. Collapsing the circle bounding each of these open discs to a point gives surjections

$$
X_{n} \rightarrow \mathbf{D}
$$

and, if we make the implied choices correctly, this will factor as

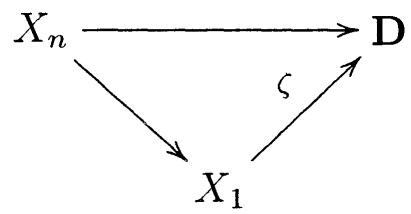

Repeated applications of Proposition 7 prove the following.

Proposition 8. If every $B$ in $\mathscr{B}$ has the property (IR) then $C\left(X_{1}\right) \rightarrow C\left(X_{n}\right)$ and $C(\mathbf{D}) \rightarrow C\left(X_{n}\right)$ are weakly corona extendible w.r.t. $\mathscr{B}$.

\section{Avoiding an Obstruction to Lifting.}

A unital $*$-homomorphism

$$
\varphi: C\left(S^{2}\right) \rightarrow Q=\prod B_{n} / \bigoplus B_{n}
$$

corresponds to a triple $\left(h_{1}, h_{2}, h_{3}\right)$ of self-adjoint elements in $Q$ that commute and for which

$$
h_{1}^{2}+h_{2}^{2}+h_{3}^{2}=1 \text {. }
$$

There is a possible $K$-theoretic obstruction to lifting $\varphi$.

It is more convenient to use the relations

$$
0 \leq h \leq 1, \quad x^{*} x=x x^{*}, \quad h x=x h \quad \text { and } \quad h^{2}+x^{*} x=h
$$

which generate the same universal, unital $C^{*}$-algebra as $(*)$, namely $C\left(S^{2}\right)$. Here we have a non-unital universal $C^{*}$-algebra as well, $C_{0}\left(\mathrm{R}^{2}\right)$. In this setting, the index of a pair $(h, x)$ in $A$, where $A$ is a unital $C^{*}$-algebra, $0 \leq h \leq 1$,

$$
\left\|x^{*} x-x x^{*}\right\| \leq \delta, \quad\|h x-x h\| \leq \delta \quad \text { and } \quad\left\|h^{2}+x^{*} x-h\right\| \leq \delta,
$$

is based on the self-adjoint matrix 


$$
P=\left[\begin{array}{cc}
h & x \\
x^{*} & 1-h
\end{array}\right] .
$$

Since $\left\|P^{2}-P\right\| \leq 2 \delta$, so long as $\delta<1 / 8$, the projection $\chi_{(1 / 2, \infty)}(P)$ will be an element of $A$. The $K$-theoretic index of the pair $(h, x)$ is defined as the $K_{0}$ class of $\chi_{(1 / 2, \infty)}(P)$ minus the class of the identity.

A more refined index is what we'll refer to as the Murray von Neumann index. This is the class of $\chi_{(1 / 2, \infty)}(P)$ among the projections of $\mathbf{M}_{2}(A)$ under Murray von Neumann equivalence. We'll say that this index is trivial if it is the same as the class of

$$
\left[\begin{array}{ll}
1 & 0 \\
0 & 0
\end{array}\right]
$$

Directly in terms of $P$, the Murray von Neumann index is trivial if and only if there exists $w \in \mathbf{M}_{2}(A)$ such that

$$
w w^{*}=\left[\begin{array}{ll}
1 & 0 \\
0 & 0
\end{array}\right]
$$

and

$$
\left\|w^{*} w-P\right\| \leq 4 \delta .
$$

The following is similar to a result (the remark following Lemma 2.3) in [Lin2], and the proof was certainly inspired by Lin's proof. Lin shows that, when an obstruction vanishes, a copy of $C\left(S^{2}\right)$ in a $C^{*}$-algebra must be contained in a larger commutative $C^{*}$-algebra. The novelty of Theorem 9 is in choosing this larger commutative algebra to have spectrum the annulus $X_{1}$. It also applies to a larger class of $C^{*}$-algebras.

Recall we are considering $S^{2}$ to be subset of $\mathrm{R} \times \mathrm{C}$,

$$
S^{2}=\left\{\left.(r, z)\left|r^{2}+\right| z\right|^{2}=r\right\}
$$

and call the coordinate functions $h$ and $x$ when regarded as elements in $C\left(S^{2}\right)$.

Theorem 9. Consider

$$
\xi: X_{1} \rightarrow S^{2}
$$

defined by $\xi\left((r+1) e^{2 \pi i \theta}\right)=\left(r, \sqrt{r-r^{2}} e^{2 \pi i \theta}\right)$ and the inclusion

$$
\eta: C\left(S^{2}\right) \rightarrow C\left(X_{1}\right)
$$

induced by $\xi$. Suppose $Q$ is a $C^{*}$-algebra in which every element of $R(Q)$ has a unitary polar decomposition. Given a unital *-homomorphism 


$$
\varphi: C\left(S^{2}\right) \rightarrow Q
$$

if there exists $w$ in $\mathbf{M}_{2}(Q)$ such that

$$
w w^{*}=\left[\begin{array}{ll}
1 & 0 \\
0 & 0
\end{array}\right]
$$

and

$$
w^{*} w=\left[\begin{array}{cc}
\varphi(h) & \varphi(x) \\
\varphi\left(x^{*}\right) & 1-\varphi(h)
\end{array}\right],
$$

then there is a unital $*$-homomorphism $\bar{\varphi}$ so that

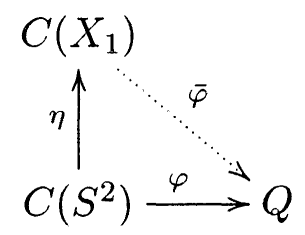

commutes.

Proof. First let us examine $\eta$ at the level of generators and relations. There is an isomorphism of $C\left(X_{1}\right)$ with

$$
C^{*}\left\langle k, u \mid 0 \leq k \leq 1, u^{-1}=u^{*}, k u=u k\right\rangle
$$

where $k$ and $u$ are concretely given as the functions

$$
k\left((r+1) e^{2 \pi i \theta}\right)=r
$$

and

$$
u\left((r+1) e^{2 \pi i \theta}\right)=e^{2 \pi i \theta} .
$$

With the specified generators and relations for $C\left(S^{2}\right)$ given above, one clearly has $\eta(h)=k$ and $\eta(x)=\sqrt{k-k^{2}} u$.

Now suppose $\varphi$ and $w$ are given as in the hypotheses of the Theorem. The condition on $w w^{*}$ forces the second row of $w$ to be zero. Therefore, we can translate the theorem as follows: we are given $h, x, a$, and $b$ in $Q$ that satisfy

$$
\begin{gathered}
0 \leq h \leq 1, \quad x^{*} x=x x^{*}, \quad h x=x h \\
h^{2}+x^{*} x=h \\
{\left[\begin{array}{ll}
a & b \\
0 & 0
\end{array}\right]\left[\begin{array}{ll}
a^{*} & 0 \\
b^{*} & 0
\end{array}\right]=\left[\begin{array}{ll}
1 & 0 \\
0 & 0
\end{array}\right]}
\end{gathered}
$$


and

$$
\left[\begin{array}{ll}
a^{*} & 0 \\
b^{*} & 0
\end{array}\right]\left[\begin{array}{ll}
a & b \\
0 & 0
\end{array}\right]=\left[\begin{array}{cc}
h & x \\
x^{*} & 1-h
\end{array}\right] .
$$

We must find a unitary $u$ in $Q$ such that $u$ commutes with $h$ and

$$
x=u \sqrt{h-h^{2}} .
$$

Since $h^{2}+x^{*} x=h$ this is the same as finding a unitary polar decomposition

$$
x=u|x|
$$

such that $u$ commutes with $h$.

If we write out the components of the matrix equations we find that

$$
\begin{aligned}
a a^{*}+b b^{*} & =1, \\
a^{*} a & =h, \\
b^{*} b & =1-h, \\
a^{*} b & =x .
\end{aligned}
$$

Notice these imply

$$
a^{*} a+b^{*} b=1 .
$$

Lemma 6(3) implies that $a^{*}$ and $b$ are in $R(Q)$ so there are unitaries $z$ and $v$ in $Q$ such that

$$
\begin{aligned}
& a=\left(a a^{*}\right)^{1 / 2} z, \\
& b=v(1-h)^{1 / 2} .
\end{aligned}
$$

It follows that

$$
1=a^{*} a+b^{*} b=z^{*}\left(a a^{*}\right) z+(1-h)
$$

and

$$
1=a a^{*}+b b^{*}=a a^{*}+v(1-h) v^{*}
$$

Therefore

$$
v h v^{*}=a a^{*}, \quad z^{*}\left(a a^{*}\right) z=h
$$

and so $u=z^{*} v$ commutes with $h$. Moreover $v h^{1 / 2} v^{*}=\left(a a^{*}\right)^{1 / 2}$ and so

$$
x=a^{*} b=z^{*}\left(a a^{*}\right)^{1 / 2} v(1-h)^{1 / 2}=z^{*} v \sqrt{h-h^{2}}=u|x| .
$$

Theorem 10. Let $\xi: X_{n} \rightarrow S^{2}$ denote the surjection that comes from collapsing each circle component of the boundary of the $n$ hole disk to a point. Let 
$\eta: C\left(S^{2}\right) \rightarrow C\left(X_{n}\right)$ denote the induced $*$-homomorphism. Let

$$
Q=\prod B_{n} / \bigoplus B_{n}
$$

where each $B$ is a unital $C^{*}$-algebra with property (IR). Given $\varphi: C\left(S^{2}\right) \rightarrow Q$, if

$$
\left[\begin{array}{cc}
\varphi(h) & \varphi(x) \\
\varphi\left(x^{*}\right) & 1-\varphi(h)
\end{array}\right]
$$

is Murray von Neumann equivalent to

$$
\left[\begin{array}{ll}
1 & 0 \\
0 & 0
\end{array}\right]
$$

then there exists $\bar{\varphi}$ so that

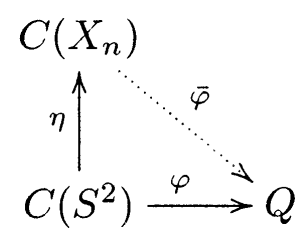

commutes.

Proof. By Lemma 6 (2), Theorem 9 applies, proving the result in the case $n=1$. The case $n=0$ follows from this directly, while for $n>1$ one may first extend to $C\left(X_{1}\right)$ and then apply Proposition 8 .

We will use the $n=1$ case of Theorem 10 to derive our main result, Theorem 12, from the Friis-Rørdam result. The more general form of Theorem 10 is used in the next section, to prove a rather unappealing corollary. The power of Corollary 11 comes from the techniques developed with Eilers and Pedersen to identify certain amalgamated products. In [ELP] very concrete examples will be given that depend on the following abstraction.

\section{A Diversion.}

Consider an open patch in $S^{2}$ that avoids the $n$ points that are the images of circles under the surjection $X_{n} \rightarrow S^{2}$. This gives us inclusions $\iota: C_{0}\left(\mathrm{R}^{2}\right) \rightarrow C\left(S^{2}\right)$ and $\iota_{n}: C_{0}\left(\mathrm{R}^{2}\right) \rightarrow C\left(X_{n}\right)$.

Suppose $\alpha: C_{0}\left(\mathrm{R}^{2}\right) \rightarrow I$ is a $*$-homomorphism, with no restriction on the $C^{*}$-algebra $I$. We can form two products

$$
P_{1}=I *_{C_{0}\left(\mathrm{R}^{2}\right)} C\left(S^{2}\right)
$$

and 


$$
P_{2}=I *_{C_{0}\left(\mathrm{R}^{2}\right)} C\left(X_{n}\right)
$$

The identity on $I$ and the inclusion induced by the surjection of $X_{n}$ onto $S^{2}$ lead to a map

$$
\theta: P_{1} \rightarrow P_{2} \text {. }
$$

Let $\iota_{\alpha}$ denote the canonical inclusion of $C_{0}\left(\mathrm{R}^{2}\right)$ into $P_{1}$ (as the algebra over which happens the amalgamation).

Lemma 11. Suppose $\mathscr{B}$ is a class of unital $C^{*}$-algebras such that each $B$ in $\mathscr{B}$ has property (IR), $K_{0}(B)$ is torsion-free and $K_{0}$ equivalence of projections in $\mathbf{M}_{2}(B)$ implies Murray-von Neumann equivalence. With $\alpha$ and $P_{i}$ as described above:

(1) If $K_{0}(I)$ is torsion then $\theta$ is corona extendible w.r.t. $\mathscr{B}$.

(2) In general, given $\varphi: P_{1} \rightarrow Q$, if the $K$-theory of $\varphi \circ \iota_{\alpha}$ is zero then there exists $\bar{\varphi}$ so that

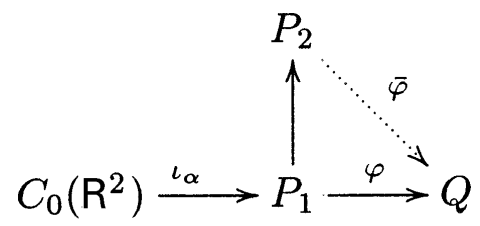

commutes, where $Q=\prod B_{n} / \bigoplus B_{n}$ for some $B_{n}$ in $\mathscr{B}$.

Proof. Given

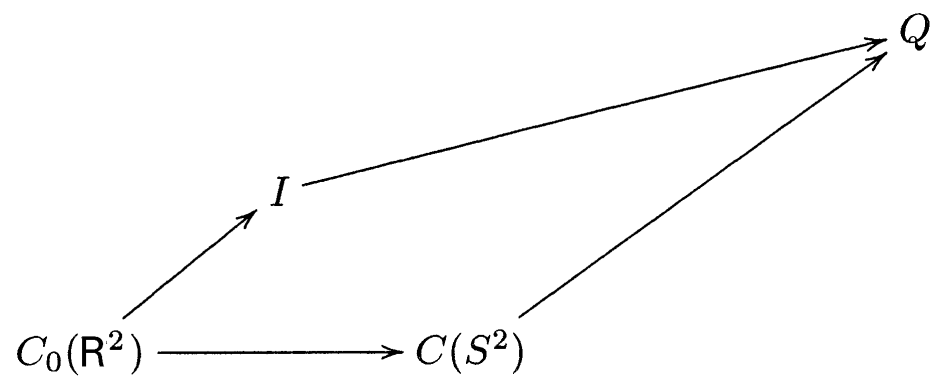

we must find a map from $C\left(X_{n}\right)$ to $Q$ so that 


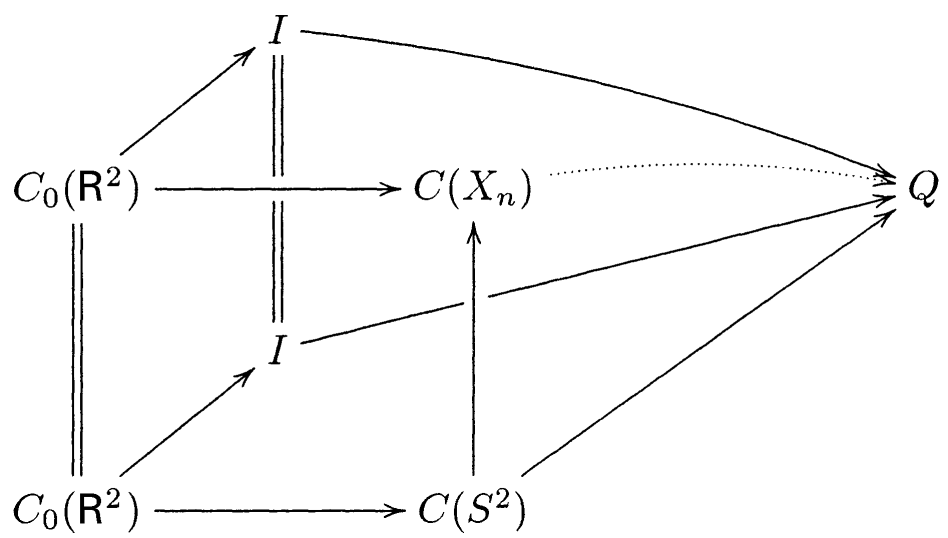

commutes. If $K_{0}(I)$ is a torsion group then this is no trouble because the map from $C_{0}(\mathrm{R})$ to $Q$ via $C\left(S^{2}\right)$ is the same as the map via $I$, and this has zero $K$ theory since $K_{0}(Q)$ is torsion free. (See [Lo1; p. 199].) The needed map comes then from Theorem 10. For (b), we can again use the theorem above because of the commutativity of the following part of the diagram, hidden before now:

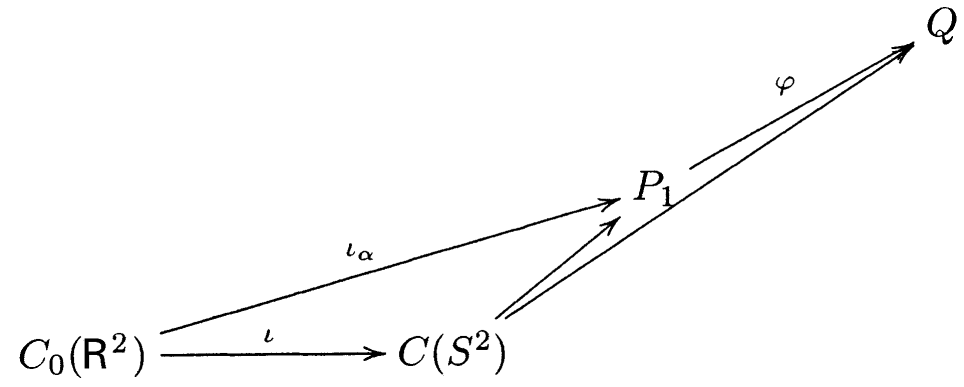

The Primary Target.

TheOrem 12. For every $\epsilon>0$ there is a $\delta>0$ so that, for every pair $(x, h)$ of elements in a $C^{*}$-algebra $B$ with property (IR) such that

$$
\|[h, x]\| \leq \delta, \quad\left\|\left[x, x^{*}\right]\right\| \leq \delta, \quad 0 \leq h \leq 1
$$

and

$$
\left\|h^{2}+x^{*} x-h\right\| \leq \delta,
$$

if the Murray von Neumann index of $(h, x)$ is trivial then there is a pair $(\bar{h}, \bar{x})$ in $B$ with

$$
\bar{h} \bar{x}=\bar{h} \bar{x}, \quad \bar{x}^{*} \bar{x}=\bar{x} \bar{x}^{*}, \quad 0 \leq \bar{h} \leq 1
$$




$$
\bar{h}^{2}+\bar{x}^{*} \bar{x}=\bar{h}
$$

and

$$
\|\bar{h}-h\|,\|\bar{x}-x\| \leq \epsilon,
$$

Proof. If false, then there is a sequence $B_{n}$ of (IR) $C^{*}$-algebras, a $*-$ homomorphism

$$
\varphi: C\left(S^{2}\right) \rightarrow Q=\prod B_{n} / \bigoplus B_{n}
$$

which does not lift to $\prod B_{n}$, and a partial isometry $w \in \mathbf{M}_{2}(Q)$ with

$$
w w^{*}=\left[\begin{array}{ll}
1 & 0 \\
0 & 0
\end{array}\right]
$$

and

$$
w^{*} w=\left[\begin{array}{cc}
\varphi(h) & \varphi(x) \\
\varphi\left(x^{*}\right) & 1-\varphi(h)
\end{array}\right] .
$$

We are now using $h$ and $x$ to again denote the coordinate functions in $C\left(S^{2}\right)$. Recall we have inclusions $C\left(S^{2}\right) \subseteq C(\mathbf{D}) \subseteq C\left(X_{1}\right)$. By Theorem 9 we can extend $\varphi$ to $C\left(X_{1}\right)$, and so by restriction there is an extension of $\varphi$ to $\bar{\varphi}: C(\mathbf{D}) \rightarrow Q$. This lifts to the product

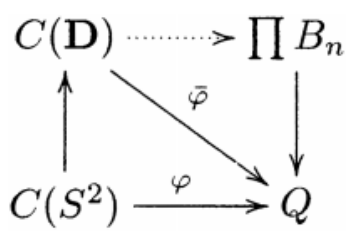

by the Friis-Rørdam extension of Theorem 1, which provides a lift of $\varphi$, a contradiction.

\section{A Corollary in Linear Algebra.}

In the special case of projections in $\mathbf{M}_{2}\left(\mathbf{M}_{d}\right)=\mathbf{M}_{2 d}$ Murray von Neumann equivalence is the same as equality of $K_{0}$ classes. Moreover, the classes correspond to the natural numbers between zero and $2 d$ as is determined by counting the multiplicity of the eigenvalue one of a projection. In this restricted, yet very important case, we can spell out Theorem 12 in very elementary terms. For this result, we use the equation $(*)$ in place of $(\dagger)$.

COROllary 13. For every $\epsilon$, there is a $\delta$ so that, for any $d$ and any self-adjoint $d$ by $d$ matrices $h_{1}, h_{2}$ and $h_{3}$ such that 


$$
\left\|h_{i} h_{j}-h_{j} h_{i}\right\| \leq \delta, \quad\left\|h_{1}^{2}+h_{2}^{2}+h_{3}^{2}-I\right\| \leq \delta
$$

if the number of positive eigenvalues of

$$
\left[\begin{array}{cc}
h_{1} & h_{2}+i h_{3} \\
h_{2}-i h_{3} & -h_{1}
\end{array}\right],
$$

counted with multiplicity, is $d$ then there are self-adjoint, commuting matrices $k_{1}, k_{2}, k_{3}$ with $k_{1}^{2}+k_{2}^{2}+k_{3}^{2}=1$ and $\left\|h_{j}-k_{j}\right\| \leq \epsilon$.

That a positive eigenvalue count is an obstruction, realized in simple examples, is well established. See [Ch], [Da] and [Lo1].

\section{Final Remarks.}

The subject of stable relations (a.k.a. semiprojectivity) has been expanding rapidly of late. The monograph [Lo3] attempts to pull together the foundation of the subject. It addresses only peripherally the question of forms of semiprojectivity conditional on the vanishing of $K$-theoretic obstructions.

Huaxin Lin has investigated semiprojectivity with respect to almost commuting elements in purely infinite, simple $C^{*}$-algebras. In the purely infinite case, one can sometimes achieve stability even when the $K$-theory indices do not vanish. As noted in [Lin3], Lin's techniques (which are rather different to those used in this paper) can be modified to give stability, conditional on the index, in the some finite, simple cases. Indeed, Corollary 13 also follows as a special case of recent results by Gong and Lin [GL].

\section{REFERENCES}

[Bl] B. Blackadar, Shape Theory for $C^{*}$-algebras, Math. Scand. 56 (1985), 249-275.

[Ch] M.-D. Choi, Almost commuting matrices need not be nearly commuting, Proc. Amer. Math. Soc. 102 (1988), 528-533.

[Da] K. R. Davidson, Almost commuting Hermitian matrices, Math. Scand. 56 (1985), 222240.

[ELP] S. Eilers, T. A. Loring and G. K. Pedersen, Morphisms of extensions of $C^{*}$-algebras: pushing forward the Busby invariant, Adv. Math. (to appear).

[FR] P. Friis and M. Rørdam, Almost commuting self-adjoint matrices-a short proof of Huaxin Lin's theorem, J. Reine Angew. Math. (to appear).

[GL] G. Gong and H. Lin, Almost multiplicative morphisms and almost commutative matrices, preprint.

[Lin1] H. Lin, Almost commuting self-adjoint matrices and applications, in Fields Institute Communications, 13 (1997), 193-234.

[Lin2] H. Lin, Homomorphisms from $C(X)$ into $C^{*}$-algebras, Canad. J. Math. to appear.

[Lin3] H. Lin, Almost commuting unitary elements in purely infinite simple $C^{*}$-algebras, Math. Ann. 303 (1995), 599-616. 
[Lo1] T. A. Loring, K-theory and almost commuting matrices, Canad. J. Math. 40 (1988) 197216.

[Lo2] T. A. Loring, Stable relations II: corona semiprojectivity and dimension-drop $C^{*}$-algebras, Pacific J. Math 172 (1996), 461-475.

[Lo3] T. A. Loring, Lifting Solutions to Perturbing Problems in $C^{*}$-Algebras, American Mathematical Society Providence, R.I. Fields Institute Monograph Series, 8 (1997).

DEPARTMENT OF MATHEMATICS AND STATISTICS

UNIVERSITY OF NEW MEXICO

ALBUQUERQUE, NM 87131

USA

e-mail: loring@math.unm.edu 